\title{
Hacia la erradicación del virus de la hepatitis C
}

\author{
Towards the eradication of hepatitis $C$ virus
}

\author{
Álvaro Mena ${ }^{1,2}$, José D Pedreira ${ }^{1,2}$ \\ ${ }^{1}$ Servicio de Medicina Interna. Complejo Hospitalario Universitario de A Coruña. SERGAS. \\ ${ }^{2}$ Grupo de Virología Clínica. Instituto de Investigación Biomédica de A Coruña (INIBIC), Complejo Hospitalario Universitario de A Coruña, SERGAS. Uni- \\ versidade da Coruña.
}

La Organización Mundial de la Salud estima que alrededor de 150 millones de personas en el Mundo están infectadas por el virus de la hepatitis C (VHC), siendo ésta la principal causa de cirrosis hepática, carcinoma hepatocelular y trasplante hepático en nuestro medio. Los pacientes con hepatopatía crónica por VHC (HCC) presentan un riesgo de mortalidad hasta 12 veces superior a la población general'1. En 2010, en Europa, las infecciones por VHC y VHB en conjunto, provocaron 10 veces más muertes que la infección por VIH, las dos terceras partes de ellas fueron debidas al $\mathrm{VHC}^{2}$.

Existen dos cuestiones fundamentales para llegar en un futuro a la hipotética erradicación del VHC, el diagnóstico y el tratamiento.

\section{Diagnóstico}

En el año 1989 un grupo de investigadores de los Centers for Disease Control and Prevention (CDC) describe por primera vez parte del material genético de las, hasta entonces, hepatitis no-A no-B ${ }^{3}$. Este mismo organismo, en 1998 establece la necesidad de realizar cribado de HCC a las personas pertenecientes a grupos de riesgo (uso parenteral de drogas, pacientes en hemodiálisis, $\mathrm{VIH}+$, receptores de trasplante de órgano o transfusiones antes de 1992), pacientes con sospecha clínica y personas con exposición reconocida. En el año 2012 añade a estas recomendaciones la necesidad del screening poblacional, una vez en la vida, a todas las personas nacidas en el periodo 1945-1965. Esta novedad parte del análisis de una gran cohorte norteamericana, la National Health and Nutrition Examinations Survey (NHANES), donde la prevalencia de infección por VHC fue 5 veces mayor en los nacidos entre 1945 y 1965 que en el resto; el 76,5\% de los infectados y el $73 \%$ de las muertes asociadas al VHC pertenecía a este grupo etario ${ }^{5}$.

La mayor prevalencia responde a distintos factores: es la generación del Baby Boom en Estados Unidos tras la II Guerra Mundial, que estuvo masivamente expuesta a procedimientos quirúrgicos y transfusiones por las distintas campañas militares en los años 60 y 70, época en la que además, se incrementó en gran medida el uso y comercialización de la heroína. Todos estos determinantes deben tenerse en cuenta cuando se pretende diseñar una estrategia de cribado adaptada a una población. Resulta apetecible implantar las recomendaciones de Estados Unidos en nuestra comunidad, pero es necesario conocer el pico de prevalencia para poder establecer una recomendación según el año de nacimiento en nuestro país. Datos recientes en población gallega muestran que el pico de prevalencia de infección por VHC se establece en los pacientes nacidos entre 1955 y $1975^{6}$. Aunque se trata de un estudio retrospectivo de todas las serologías realizadas en un área sanitaria y no propiamente de cribado poblacional, en el rango de pacientes nacidos entre 1965 y 1975 se diagnosticaron el 32,7\% de los pacientes, grupo que queda fuera de las recomendaciones norteamericanas; hasta el momento no hay más datos publicados en esta línea en España. De nuevo, la Historia nos permite explicar estas diferencias: no hay un claro Baby Boom tras la Guerra Civil Española, sino que la natalidad presenta un progresivo aumento a partir de los años 50 y hasta 1979; 0 la epidemia de drogadicción parenteral en España es posterior a Estados Unidos u otros países europeos.

Se estima que en los países desarrollados la mitad de los pacientes con HCC están sin diagnosticar (43,1\% en EEUU), por tanto, el diagnóstico es pieza clave si se pretende erradicar el $\mathrm{VHC}^{7}$.

\section{Tratamiento}

En el año 1991 la FDA aprueba el primer tratamiento con interferón a. En 1998 se inician de las terapias combinadas con interferón (IFN) y ribavirina (RBV), lo que supuso una mejora en la eficacia, sobre todo en los genotipos con mejor respuesta (Genotipo 2 y 3) sin añadir excesivos efectos adversos, a los ya frecuentes con una terapia con IFN ${ }^{8}$. Posteriormente se desarrollaron los interferones pegilados (PegIFN), en uso desde $2001^{9}$, que aumentaron la eficacia del tratamiento con una ligera disminución de los efectos secundarios, aunque continuaban presentes hasta en el $100 \%$ de los enfermos y eran especialmente limitantes en algunas poblaciones especiales (cirróticos severos, trasplantados hepáticos y $\mathrm{VIH}+$ ), pacientes que, además, presentaban menores tasas de curación y mayor urgencia terapéutica. Se diseñó un esquema de tratamiento estándar de 48 semanas que, en ocasiones se prolongaba a 72 semanas 0 se acortaba a 24, según el genotipo y la respuesta viral.

Los primeros antivirales de acción directa (AAD), boceprevir y telaprevir, se comercializaron en España a partir de 2012, junto con las recomendaciones por parte de la Agencia Española de Medicamentos y Productos Sanitarios, posteriormente todas las Comunidades Autónomas fueron matizando este documento, de manera más o menos restrictiva. Estos primeros $A A D$ tienen la indicación en combinación con PegIFN y RBV para el tratamiento únicamente del genotipo 1, añaden eficacia tanto en pacientes naive, donde las tasas de respuesta pasan del $50 \%$ al $75 \%$, y en 


\section{“...seremos capaces de erradicar el VHC en los próximos años y reducir el número de complicaciones asociadas únicamente si establecemos estrategias de cribado y tratamiento activas, efi- cientes y adaptadas a nuestra población"}

pretratados, sobre todo los pacientes respondedores con recaída. Aparecen nuevos conceptos como las reglas de parada, donde una mala respuesta al tratamiento en las primeras semanas obliga a la suspensión del mismo, minimizando los efectos adversos, evitando el riesgo de resistencias emergentes y suponiendo un ahorro en pacientes con nulas posibilidades de curación. Uno de sus puntos débiles es el considerable aumento en los efectos secundarios, exacerbando los existentes (anemia, trombopenia, astenia. . . ) o apareciendo otros propios (toxicodermia, disgeusia, entre otros). Esta toxicidad es aún más intensa y limitante en las poblaciones especiales mencionadas previamente, que conlleva a peores tasas de respuesta. Supone una mejoría en el tratamiento pero existen algunas limitaciones: solo para genotipo 1, baja respuesta en pacientes respondedores parciales o nulos, alta tasa de efectos adversos (y en ocasiones graves) en pacientes con más necesidad de tratamiento (poblaciones especiales), uso únicamente con PegIFN, alto número de comprimidos diarios (6 para telaprevir y 12 para boceprevir, más la RBV y el PegIFN), tratamientos complejos para el enfermo (con impacto en su vida personal y profesional) y para el equipo asistencial (numerosas visitas, determinaciones microbiológicas en plazos de 24/48h, frecuentes consultas no planificadas, monitorización estrecha de la adherencia y medicaciones concomitantes por interacciones. . .). Recientemente se ha comercializado en España, la siguiente oleada de AAD: simeprevir y sofosbuvir. Suponen una nueva revolución en el tratamiento del VHC con un doble uso, añadidos a PegIFN y RBV o combinados entre sí en terapias libres de PegIFN. En el primer escenario, permiten tratamientos más cortos (12-24 semanas), de más genotipos (1 y 4 en el caso de simeprevir y pangenotípico sofosbuvir), con 1 comprimido/ día, muy pocos efectos secundarios propios (presentes los del PegIFN y RBV), y tasas de curación cercanas al $90 \%$ en pacientes naive y respondedores con recaída y superiores al $50 \%$ en respondedores parciales y nulos. Pero el escenario ideal para estos fármacos, y para los de autorización en los próximos meses, es su uso en terapias libres de PegIFN, donde con pautas sencillas (2-3 comp/día \pm RBV), sin efectos secundarios y con tratamientos de 8-12 semanas, se consigue curar al 100\% de los pacientes. Con estos fármacos ya no hay poblaciones especiales (la respuesta en cirróticos severos, trasplantados 0 $\mathrm{VIH}+$ es también cercana al $100 \%$ ), no hay genotipos favorables o desfavorables; por su alta eficacia, los factores predictores de respuesta clásicos pierden su capacidad predictiva (probablemente se desarrollarán más estrategias moleculares con este

Figura 1. Gasto en distintas especialidades farmacéuticas en España durante 2013.

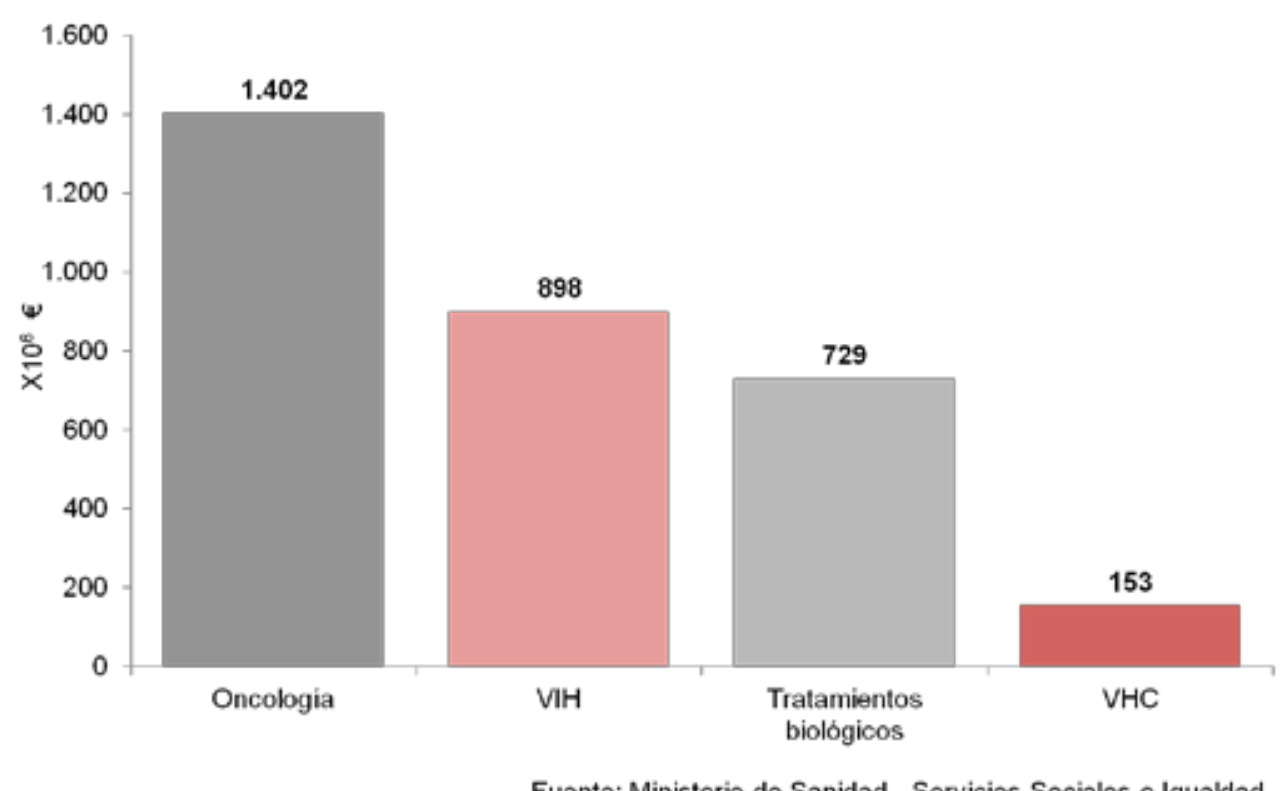


fin), no hay suspensiones de tratamientos por efectos adversos ni mayor complejidad asistencial por los mismos.

El precio es el factor limitante para el acceso masivo a estas terapias, lo cual ha demorado su comercialización y ha llevado a las empresas farmacéuticas a asumir diferentes acuerdos de sostenibilidad y a la comercialización de los fármacos a precios sensiblemente inferiores a otros países $(84.000 \$$ para 12 semanas de sofosbuvir $066.000 \$$ de simeprevir en EEUU, frente a los menos de 25.000 de ambos en España). Si bien es cierto que su precio es elevado, hay que tener en cuenta el impacto de estos tratamientos en el gasto en farmacia global en España; en la Figura1 se muestra el gasto en 2013 en distintas familias de fármacos, año donde los primeros AADs (boceprevir y telaprevir) ya estaban comercializados, y a precios similares a Ios nuevos AADs. No hay que olvidar que, un paciente cirrótico descompensado o con un hepatocarcinoma consume más de 6.000 /año 0 el coste del primer año del trasplante hepático ronda los $65.000{ }^{10}$.

Un artículo británico recientemente publicado pretende estimar el número de hepatocarcinomas y muertes secundarias a HCC en el año 2020 si se continúa con el escenario diagnóstico y terapéutico de 20130 se incrementan ambos. En la situación de 2013 los hepatocarcinomas y las muertes asociadas a la HCC aumentarán aproximadamente el $50 \%$ en 2020 y el $100 \%$ en 2030. Tan solo con un incremento del $50 \%$ de pacientes diagnosticados y de pacientes tratados en los próximos años es posible reducir en un 30\% estas complicaciones en $2020^{11}$. Como conclusión, seremos capaces de erradicar el VHC en los próximos años y reducir el número de complicaciones asociadas únicamente si establecemos estrategias de cribado y tratamiento activas, eficientes y adaptadas a nuestra población. Por tanto, es tiempo de diagnosticar, tratar y curar a los pacientes con HCC.

\section{Bibliografía}

1. Mahajan R, Xing J, Liu SJ, Ly KN, Moorman AC, et al. Mortality Among Persons in Care With Hepatitis C Virus Infection: The Chronic Hepatitis Cohort Study (CHeCS), 2006-2010. Clin Infect Dis 2014;58:1055-1061.

2. Negro F. Facts and fictions of HCV and comorbidities: Steatosis, diabetes mellitus, and cardiovascular diseases. Journal of Hepatology 2014;61:S69-S78.

3. Choo Q-L, Kuo G, Weiner AJ, Overby LR, Bradley DW, Houghton M. Isolation of a cDNA clone derived from a blood-borne non-A, non-B viral hepatitis genome. Science 1989;244:359-362.

4. CDC. Recommendations for the identification of chronic hepatitis C virus (HCV) among persons born during 1945-1965. MMWR Recomm Rep 2012;61(RR-4):132.

5. Denniston MM, Klevens RM, McQuillan GM, Jiles RB. Awareness of infection, knowledge of hepatitis $\mathrm{C}$, and medical follow-up among individuals testing positive for hepatitis C: National Health and Nutrition Examination Survey 2001-2008. Hepatology 2012;55:1652-1661.

6. Mena A, Moldes L, Meijide H, Cañizares A, Castro-Iglesias A, Delgado M, et al. Seroprevalence of HCV and HIV infections by Year of Birth in Spain: Impact of US CDC and USPSTF Recommendations for HCV and HIV Testing. PLoS One. 2014 DeC 1;9(12):e113062.

7. Spradling PR, Rupp L, Moorman AC, Lu M, Teshale EH, Gordon SC, et al. Hepatitis $B$ and $C$ virus infection among 1.2 million persons with access to care: factors associated with testing and infection prevalence. Clin Infect Dis 2012;55:1047-1055.

8. Bodenheimer HC, Lindsay KL, Davis GL, Lewis JH, Thung SN, Seeff LB. Tolerance and efficacy of oral ribavirin treatment of chronic hepatitis C: a multicenter trial. Hepatology 1997;26:473-477.

9. Zeuzem S, Feinman SV, Rasenack J, Heathcote EJ, Lai MY, Gane E, et al. Peginterferon alfa-2a in patients with chronic hepatitis C. N Engl J Med 2000;343:16661673.

10. Turnes J, Romero-Gómez M, Planas R, Solàd R, García-Samaniego J, Diago M, et al. Pharmacoeconomic analysis of the treatment of chronic hepatitisC with peginterferon alfa-2a or peginterferon alfa-2b plusribavirin in Spain. Gastroenterol Hepatol 2013;36:555-564

11. Cramp ME, Rosenberg WM, Ryder SD, Blach S, Parkes J. Modelling the impact of improving screening and treatment of chronic hepatitis $C$ virus infection on future hepatocellular carcinoma rates and liver-related mortality. BMC Gastroenterol 2014;14:137-147. 